\title{
Approaches to achieve surface sensitivity in the in situ XAS of electrocatalysts
}

\author{
Haoliang Huang and Andrea E. Russell*
}

School of Chemistry, University of Southampton, Highfield, Southampton, SO17 1BJ

\begin{abstract}
In situ and operando techniques providing information regarding adsorbate bonding and atomic arrangements on the electrode surface along with pure electrochemical measurements are needed to more fully understand the detailed mechanism of electrocatalytic reactions on high surface area/nanoparticle electrocatalysts. X-ray adsorption spectroscopy (XAS) is a powerful tool to interrogate the electronic structure and local coordination environment of such electrocatalysts under working conditions, but it should be acknowledged that standard XAS methods are not intrinsically surface sensitive. This review will present recent in situ XAS studies on single-atom, metal, and metal oxide electrocatalysts, highlighting the approaches taken to achieve surface sensitivity by careful design of the sample under investigation.
\end{abstract}

\section{Keywords}

In situ XAS, electrocatalytic reactions, surface sensitivity, single-atom electrocatalyst, nanoparticles, thin films. 


\section{Introduction}

Electrocatalysis is at the heart of electrochemical energy conversion devices, such as fuel cells and water electrolysers. Understanding the detailed mechanism of electrocatalytic reactions will provide direction that will enable the design of electrocatalysts with improved activity, stability and selectivity. Electrocatalytic reactions involve adsorption of the reactants and/or intermediates on the electrode surface and electron transfer between these species and the electrode. Although the thermodynamics and kinetics of the electron transfer step can be analysed by electrochemical measurements, such measurements need to be supplemented by complimentary techniques that provide molecular-level information regarding the bonding between the adsorbate and the surface or structural information regarding the atomic arrangement and electronic structure of the surface atoms.

X-ray absorption spectroscopy (XAS) is one such complimentary technique that has found increasing use in the study of electrocatalysts[1, 2]. The XAS spectrum can be divided into three regions as depicted in Figure 1A. At the absorption edge the energy of the incident X-ray is high enough to excite core-level electrons of an absorbing atom to unoccupied states (Figure 1A) and, from approximately $50 \mathrm{eV}$ above the edge, into the continuum producing photoelectrons, which can be scattered back by the neighbouring atoms. The outgoing photoelectron wave and the backscattered wave interfere producing the oscillation of the absorbance or fluorescence signal above the edge (Figure 1A). Arising from their physical origins, the X-ray absorption near edge (XANES) region is sensitive to the oxidation state and electronic structure of the absorber, whilst the extended X-ray absorption fine structure (EXAFS) offers information regarding local coordination of the absorber; the identity and coordination number of the neighbouring atoms and the distance between the absorber and the neighbours. XAS has two unique advantages over other techniques in characterising electrocatalysts. The first is the capability to probe the local structure regardless of the degree of crystallinity of the material, which is of particular importance for electrocatalysts as they are commonly nanoparticles, highly disordered structures, or atomically dispersed metal atoms. Secondly, XAS measurements can be conducted under electrochemical control (in situ measurements) or in an environment very similar to that of the working device (operando measurements). 

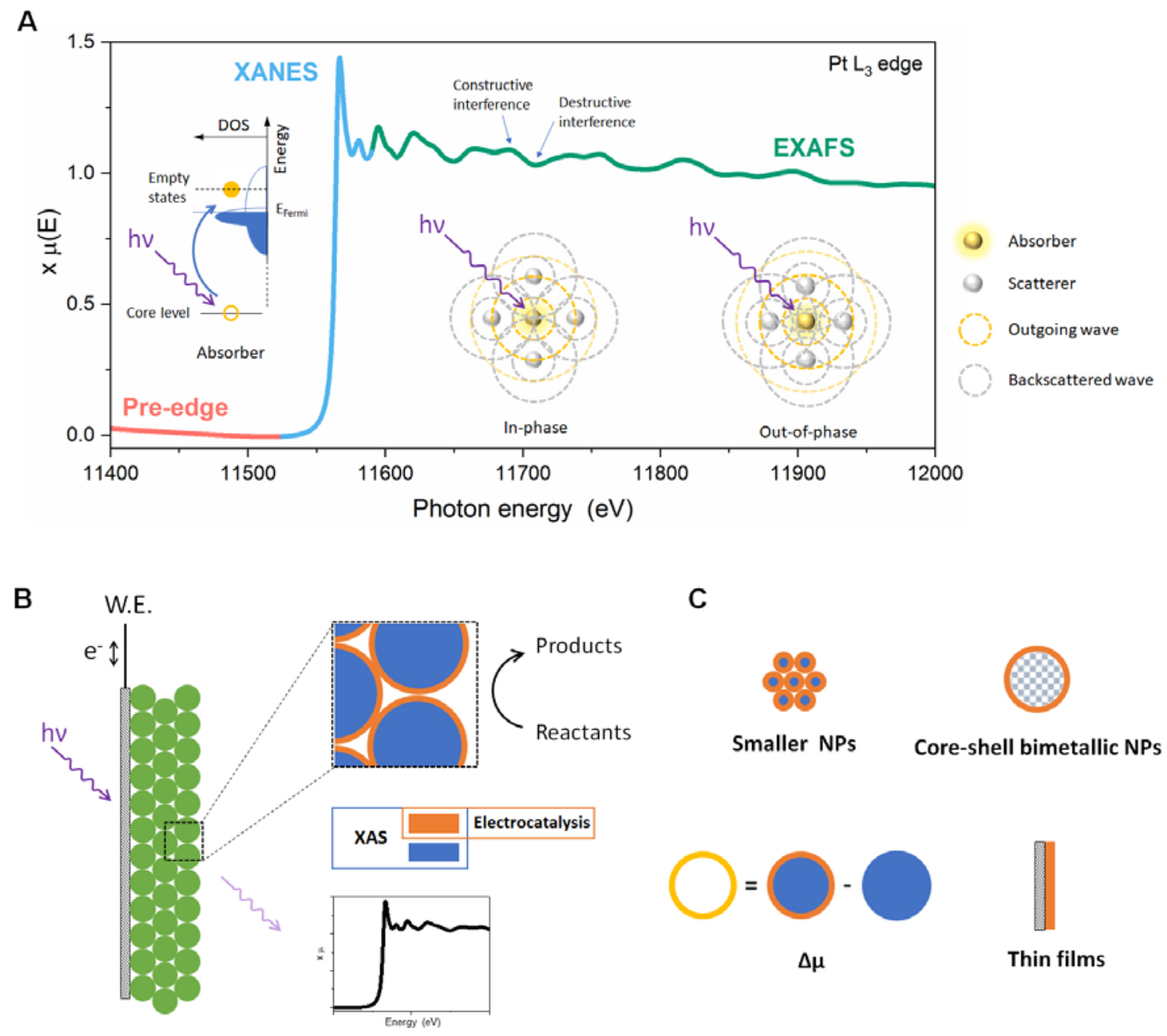

Figure 1 (A) A XAS spectrum of Pt foil at the Pt $\mathrm{L}_{3}$ edge, showing an XAS spectrum, which consists of three parts, the pre-edge (red), the XANES (cyan) and the EXAFS (green). The insets show the electron transition happening in the XANES, and the interference between photoelectrons and backscattered electrons in the EXAFS. Schematic illustration of (B) in situ XAS measurements under potential control, showing that XAS is a bulk per-atom averaging technique whilst electrochemical measurements are a probe of the surface (including the near-surface, few nanometers beneath the topmost layer, depending on the reaction), and (C) appoaches to achieve surface-sensitive XAS for electrocatalysts.

Although a wealth of information becomes available when coupling in situ or operando XAS with electrochemical measurements, the challenges should be acknowledged; the greatest of which is that the XAS and electrochemical measurements may not probe the same parts (atoms) of the electrocatalyst. The electrochemical measurements are inherently a probe of the surface (including 
the near-surface, depending on the reaction), whilst XAS is a bulk per-atom averaging technique measuring not only the surface active sites but also the spectator part in the core (Figure 1B). In addition, XAS measurements usually require higher loading or thicker electrocatalyst layers than thin films drop-cast for conventional electrochemical studies, which may compromise the conductivity of the electrocatalyst layer and the contact with electrolyte and/or reactants. Thus, sample thickness in such measurements must strike a balance between the XAS signal intensity and the electrochemistry. For transmission XAS experiments the ideal sample thickness would provide a change in absorption of approximately 1 going over the edge, whilst ensuring that the total absorbance by the sample is less than approximately 2.5 to avoid non-linear effects and, for fluorescence, self-absorption effects, that would distort the XAS data. Simultaneously the electrochemistry requires that all of the sample is electrochemically active and accessible. This can be confirmed by ensuring that the electrochemical response (active surface area or limiting currents observed) remain proportional to the sample thickness or electrode loading and have not exceeded a saturation point.

The surface sensitivity of XAS can be achieved using instrumental and sample-based approaches. The former take advantage of measuring modes using electron yield [3, 4] or by using grazing incidence geometry $[5,6]$. Compared to standard transmission/fluorescence XAS measurements, these two modes require additional complication in the cell design for in situ and operando measurements and, for the cases of high surface area or nanoparticle catalysts addressed in this review they provide less surface sensitivity as the typical diameter of the particles is close to the probing depths. In this brief review, we have thus emphasised studies that take the latter approach and seek to achieve surface and near-surface sensitivity by careful design of the sample under investigation (Figure 1C), presenting some recent in situ XAS studies to showcase the versatile and essential role of XAS measurements in understanding electrocatalytic reaction mechanisms on single-atom, metal and metal oxide electrocatalysts.

\section{Single-atom electrocatalysts}

Singe-atom electrocatalysts (SAEC), where metal atoms are atomically dispersed on suitable supporting materials, have emerged as a new branch of electrocatalytic materials as they maximise the atomic efficiency of the metal active site and enable unique atomic coordination-activity relationships [7-9]. For such electrocatalysts the electrochemistry and XAS should inherently probe the same metal atoms, enabling valuable insights into the nature of the active sites to be obtained as illustrated in the examples described below. However, as mentioned in the introduction, 
the thickness of the electrocatalyst layer must be optimised to ensure both a good XAS signal, but more importantly that all of the metal atoms are electrochemically active, which can be confirmed from the electrochemical measurements.

Among currently available SAEC, Fe atoms embedded in nitrogen-doped carbon matrices (Fe-NC) are considered as a promising non-Pt electrocatalysts for the oxygen reduction reaction (ORR). To understand how the Fe active sites behave during the ORR, Mukerjee's group [10-15] used in situ XAS on Fe-N-C catalysts prepared by pyrolysis of different precursors, and Osmieri et al.[16] recently extended the measurements to the membrane electrode assembly (MEA) level. They directly observed spectral changes corresponding to $\mathrm{Fe}^{2+} / \mathrm{Fe}^{3+}$ redox (Figure 2A) and the simultaneous addition of one oxygen neighbour. The redox transition of the Fe active site was attributed to a $\mathrm{Fe}^{2+}-\mathrm{N}_{4}$ to $(\mathrm{H}) \mathrm{O}-\mathrm{Fe}^{3+}-\mathrm{N}_{4}$ transition, of which the $\mathrm{O}(\mathrm{H})$ can be from water activation or from ORR, with $\mathrm{Fe}^{2+}-\mathrm{N}_{4}$ considered as the active site for ORR. Using this notion, the fraction of active sites as a function of potential was obtained from both the electrochemical and XANES analyses (Figure 2B) and found to be consistent with the kinetics-controlled part of ORR polarisation curves [15]. Further inspection of the average Fe-N bond length $\left(\mathrm{R}_{\mathrm{Fe}-\mathrm{N}}\right)$ by Mukerjee's group revealed that the geometry of the $\mathrm{Fe}^{2+}-\mathrm{N}_{4}$ active site can be either square-planar or out-ofplane, both of which switched to the other geometry with the $\mathrm{Fe}^{2+} / \mathrm{Fe}^{3+}$ redox and the addition of an oxygen neighbour[12, 13]. This opposite structure-switching behaviour was used to explain the differences in $\mathrm{Fe}^{3+}-\mathrm{O}$ binding, which results in the differences in the redox potential and ORR activity[15].

The active sites of Fe-N-C are not limited to the isolated $\mathrm{Fe}-\mathrm{N}_{4}$ moieties with pyridinic $\mathrm{N}$ ligands. Zhang et al.[17] recently reported an efficient Fe-N-C ORR electrocatalyst containing an $\mathrm{Fe}_{2} \mathrm{~N}_{6}$ active site fused from two Fe- $\mathrm{N}_{4}$ sites, the coordination of which was suggested by $\sim 0.8 \mathrm{Fe}$ neighbours in the first coordination shell found by analysing the Fe K-edge EXAFS data. In addition to the redox transition of $\mathrm{Fe}^{2+} / \mathrm{Fe}^{3+}$, in situ XAS suggested contractions of the $\mathrm{Fe}-\mathrm{Fe}$ distance, $\mathrm{R}_{\mathrm{Fe}-\mathrm{Fe}}$, at ORR potentials (2.18 2.19 $\AA$ ) with respect to that measured ex situ ( 2.47 $\AA$ ) and at open circuit potential $(\sim 2.23 \AA$ ), which was attributed to the dual-side adsorption of oxygenated intermediates (such as *OOH). Gu et al.[18] studied a $\mathrm{Fe}^{3+}-\mathrm{N}-\mathrm{C}$ with pyrrolic $\mathrm{N}$ ligands and using in situ XAS measurements in $0.5 \mathrm{M} \mathrm{KHCO}_{3}(\mathrm{pH}=\sim 8)$ showed that the $\mathrm{Fe}^{3+}$-N-C cation was retained at potentials as low as $-0.5 \mathrm{~V}$, in contrast to the $\mathrm{Fe}^{3+}-\mathrm{N}_{4}$ species found with pyridinic $\mathrm{N}$ ligands, which was reduced at potentials $<-0.1 \mathrm{~V}$ (spectra $>-0.1 \mathrm{~V}$ were not measured). Together with the $\mathrm{Fe}^{3+}$ to $\mathrm{Fe}^{2+}$ transition, the $\mathrm{Fe}$ centre of the $\mathrm{Fe}^{3+}-\mathrm{N}-\mathrm{C}$ was found to lose one $\mathrm{N}$ or $\mathrm{C}$ neighbour. 
Potential dependent XAS spectra have also been reported for SAEC with other metal centres. Shang et al.[19] observed dynamic Mn $\mathrm{K}$ edge XAS spectra of a $\mathrm{Mn}^{3+}-\mathrm{N}_{2} \mathrm{C}_{2}$ SAEC at potentials corresponding to the ORR (0.76-1.04 V) and oxygen evolution reaction (OER, 1.48-1.70 V) in $\mathrm{O}_{2}$ saturated $0.1 \mathrm{M} \mathrm{KOH}$ solution. The average oxidation states of $\mathrm{Mn}$, estimated from reference compounds, were found to reduce from 2.9 to 2.2 when driving ORR and to increase from 3.1 to 3.8 when driving OER, along with the elongation and the contraction of Mn-C/Mn-N by $0.04 \AA$, respectively (Figure 2C and Figure 2D). However, apart from this breathing of the bond distance no other changes, such as the coordination number of $\mathrm{Mn}$, were observed as a function of the applied potential. Redox transitions accompanied by intense structural changes of a SAEC under working condition were found for copper(II) phthalocyanine (CuPc) $\mathrm{CO}_{2}$ reduction electrocatalysts [20]. As evidenced by the characteristic pre-peak and XANES features of the Cu K-edge spectra and their derivatives, $\mathrm{CuPc}$ was reduced to $\mathrm{Cu}(\mathrm{I})$ at $-0.66 \mathrm{~V}$ and $\mathrm{Cu}(0)$ at potentials $<-0.86 \mathrm{~V}$ in $\mathrm{CO}_{2}$ saturated $0.5 \mathrm{M} \mathrm{KHCO}_{3}$ solution, and the reduction could be reversed, forming CuPc back at $0.64 \mathrm{~V}$ (Figure 2E-G). The formation of metallic $\mathrm{Cu}$ was also indicated by a $\mathrm{Cu}-\mathrm{Cu}$ scattering path ( $\mathrm{R}=\sim 2.53 \AA$ ), the coordination number (CN) of which increased as the applied potential was made more negative (Figure $2 \mathrm{H}$ ). After the correction for the 20\% unreactive CuPc present, obtained from linear combination fitting, the $\mathrm{CN}$ of the $\mathrm{Cu}-\mathrm{Cu}$ bond at $-1.06 \mathrm{~V}$ was found to correspond to $\mathrm{Cu}$ clusters with an average size of $2 \pm 1 \mathrm{~nm}$ using the cuboctahedral model. Given the similar trend of the Faradaic efficiency of $\mathrm{CH}_{4}$ product and the formation of $\mathrm{Cu}$ clusters as a function of applied potential, the metallic $\mathrm{Cu}$ clusters were suggested as the active species for $\mathrm{CO}_{2}$ to $\mathrm{CH}_{4}$ conversion. The formation of $\mathrm{Cu}$ nanoparticles during $\mathrm{CO}_{2}$ reduction was supported by in situ XAS studies on Cu-based metal/covalent organic frameworks[20, 21] and Cu-doped carbon[22, 23], with the particle size and the transition potential varied with the $\mathrm{Cu}$ contained precursors. 

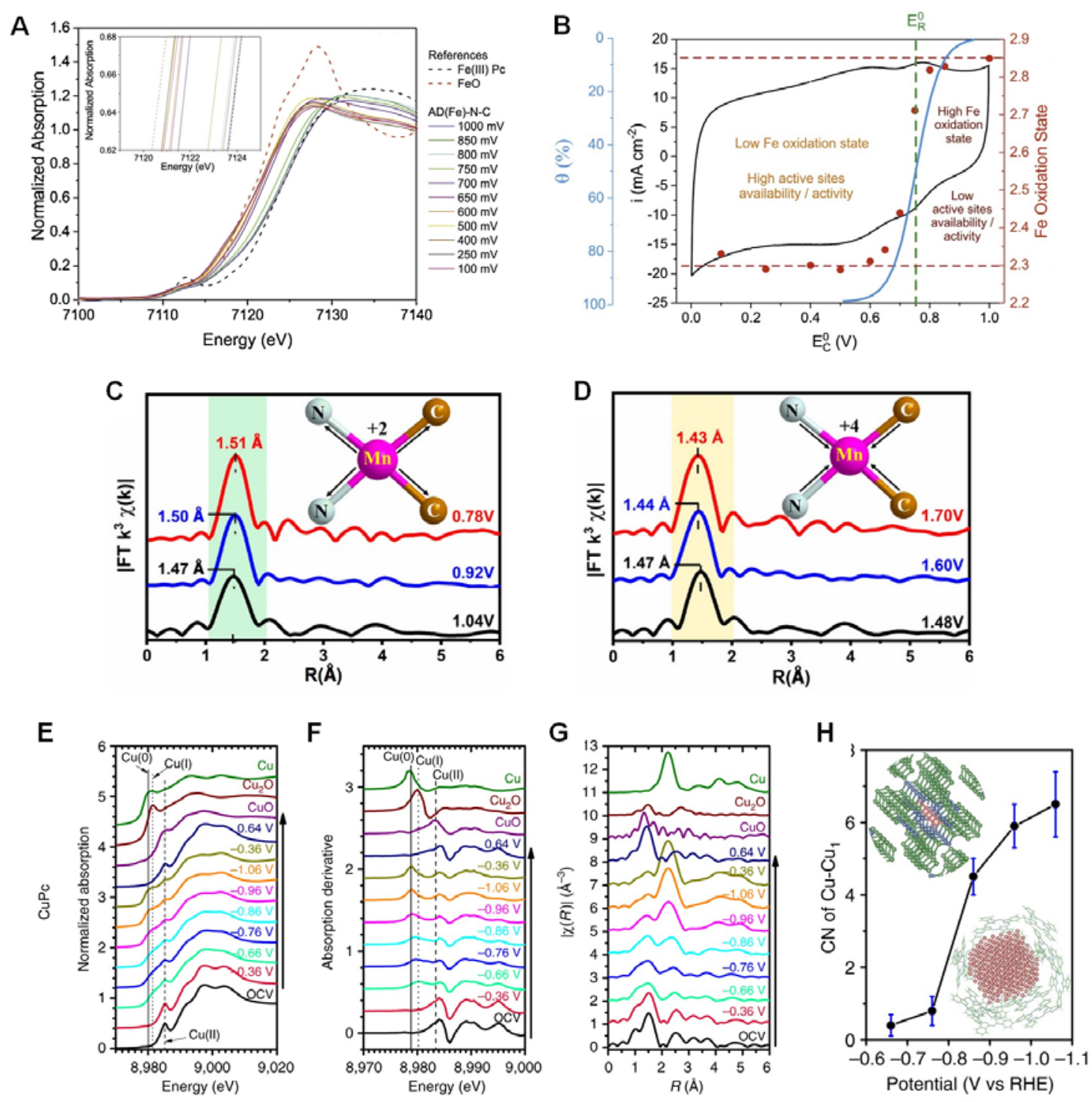

Figure 2 (A and B) In situ Fe K edge XAS measurements of an Fe-N-C catalyst. (A) XANES spectra of an Fe-N-C MEA electrode at cathode potentials of $0.1-1.0 \mathrm{~V}$. The MEA electrode was measured at $80{ }^{\circ} \mathrm{C}, 100 \% \mathrm{RH}, 1.5 \mathrm{~atm} \mathrm{with} \mathrm{H}_{2} / \mathrm{He}$ flows at the anode/cathode, respectively. (B) Fe oxidation states (red) as a function of the cathode potential ( $\mathrm{E}_{\mathrm{c}}{ }^{0}$ ), obtained from linear combination fitting using the reference samples shown in A. CV (black) of cathode at $20 \mathrm{mV} \mathrm{s}^{-1}$, showing the redox potential $\left(\mathrm{ER}_{\mathrm{R}}{ }^{0}\right)$ of the Fe-N-C MEA $(0.787 \mathrm{~V})$. Theoretical fraction of active site $(\theta$, blue), obtained from Nernst equation. Adapted from [16], Copyright (2019), with permission from Elsevier. (C and D) in situ Mn K edge EXAFS spectra of a Mn- $\mathrm{N}_{2} \mathrm{C}_{2}$ SAEC during (C) ORR and (D) OER in $\mathrm{O}_{2}$ saturated $0.1 \mathrm{M} \mathrm{KOH}$ solution, showing the elongation of $\mathrm{Mn}^{2+}-\mathrm{N} / \mathrm{C}$ bonds when driving ORR and the contraction of $\mathrm{Mn}^{4+}-\mathrm{N} / \mathrm{C}$ bonds when driving OER. Reproduced with permission from [19]. Copyright (2020) American Chemical Society. (E-H) In situ Cu K edge XAS measurements of CuPc with applied potentials in $\mathrm{CO}_{2}$ saturated $0.5 \mathrm{M} \mathrm{KHCO}_{3}$ solution, showing in (E) XANES spectra, (F) the first derivative of the XANES spectra, and (G) Fourier-transformed EXAFS spectra. (H) The CN of the first-shell $\mathrm{Cu}-\mathrm{Cu}$ plotted as a function of potentials. The inset illustrates a possible configuration of the $\mathrm{Cu}$ clusters formed under $\mathrm{CO}_{2}$ 
reduction condition and oxidized back into CuPc at $0.64 \mathrm{~V}$. Reproduced from the work of Weng et al.[20], which is licensed under Creative Commons Licence 4.0 CC BY.

\section{Nanoparticles - maximising the surface atom fraction}

Metallic, especially Pt-based, particles are of particular importance for low temperature fuel cell catalysts and in situ XAS has been used extensively to study these electrocatalysts[24]. The challenge of in situ XAS studies on metallic particles is, as shown in the introduction, that the atoms in the core dilute the XAS signal from surface atoms, which are of electrochemical interest. This dilution may blur any potential- and adsorption-induced changes of the spectra and introduce large uncertainty to determine the structural parameters of adsorbed species. The key to solve this challenge is to increase the surface-to-bulk ratio to have sufficient surface signal, which aligns well with the general requirement for practical electrocatalysts.

One of the strategies is to decrease the size of nanoparticles, normally to less than $3 \mathrm{~nm}$ corresponding to $>50 \%$ atoms on the surface (Pt as an example[25]). Imai et al. [26] studied the oxidation of $\sim 2 \mathrm{~nm}$ Pt nanoparticles ( $\sim 60 \%$ surface atoms) at $1.4 \mathrm{~V}$ in $0.5 \mathrm{M} \mathrm{H}_{2} \mathrm{SO}_{4}$ using timeresolved XAS. Fits of the EXAFS spectra suggested a gradual development of oxygen coordinated Pt atoms; at the initial stage $\mathrm{OH}_{\mathrm{ads}} / \mathrm{H}_{2} \mathrm{O}_{\text {ads }}$ with a long Pt-O bond $(\mathrm{R}=2.2-2.3 \AA$ ) was the dominant species, this species was then oxidized into $\alpha-\mathrm{PtO}_{2}$-like $2 \mathrm{D}$ oxides with a shorter Pt-O bond of $\sim 2.0$ $\AA ̊$ and a new Pt-Pt scattering path at $3.1 \AA$, and finally the $2 \mathrm{D}$ oxides were transformed into $\beta-\mathrm{PtO}_{2}-$ like 3D oxides with an additional Pt-Pt scattering path at $\sim 3.5 \AA$. Similar development of Pt oxide formation was also found by Merte et al.[27] on $~ 1.2 \mathrm{~nm}$ Pt nanoparticles ( 80\% surface atoms) with applied potentials from $-0.04 \mathrm{~V}$ to $1.26 \mathrm{~V}$ using linear combination fitting of high-energyresolution fluorescence-detected (HERFD) XANES spectra.

For nanoparticles with size larger than $5 \mathrm{~nm}$, the electrochemistry-induced changes in the XAS spectra usually become subtle. $\Delta \mu$ methods, in which the bulk metal-metal interactions are removed by subtracting spectra at a reference potential from that at the potential of interest, are used to emphasise the changes[28, 29]. Jia et al.[30] compared the adsorption of oxygenated species on Pt of octahedral Mo-PtNi nanoparticles with that of PtNi nanoparticles ( $5.5 \mathrm{~nm}$ for both samples). Whilst differences in the white line region of the $\mathrm{Pt}_{3}$ XANES were subtle, the $\Delta \mu$ method highlighted effects of the Mo ad-atoms on the potential-dependent trend of oxygen coverage on Pt. Li et al.[31] studied the existence of surface adsorbed $\mathrm{OH}\left(\mathrm{OH}_{\mathrm{ads}}\right)$ on $\mathrm{Ru} / \mathrm{C}(\sim 8 \mathrm{~nm})$ and $\mathrm{PtRu} / \mathrm{C}$ in the hydrogen oxidation region. They found that the experimental $\Delta \mu$, obtained by subtracting the XANES at $0.05 \mathrm{~V}$, where the surface was covered by adsorbed $\mathrm{H}\left(\mathrm{H}_{\mathrm{ads}}\right)$, from that at $0.24 \mathrm{~V}$, where 
$\mathrm{CO}$ stripping experiments suggested that $\mathrm{OH}_{\text {ads }}$ existed, matched with a theoretical $\Delta \mu$ obtained from FEFF calculations modelling the replacement of $\mathrm{H}_{\mathrm{ads}}$ on top of a $\mathrm{Ru}_{6}$ cluster by an $\mathrm{OH}_{\mathrm{ads}}$ (Figure 3A-C). Similarly, $\Delta$-EXAFS has also been used to detect adsorbate-induced changes on the surface of metallic particles [32, 33], the precision of which can be significantly enhanced using a modulation excitation method [34-36], which can be used for XAS under electrochemical conditions. In the modulation excitation method, any external stimulus (e.g. atmosphere, temperature or applied potential) that can induce structural changes is changed periodically with a frequency $\omega$, while spectra are collected, and parts of the measured spectra that do not follow $\omega$ (e.g. spectator species and noise) can be cancelled out using a mathematical procedure [34].

Reducing particle size to maximise surface area has also been applied to the study of metal oxide electrocatalysts. Fabbri et al.[37] prepared $\mathrm{Ba}_{0.5} \mathrm{Sr}_{0.5} \mathrm{Co}_{0.5} \mathrm{Fe}_{0.2} \mathrm{O}_{3-\delta}$ (BSCF) perovskite nanoparticles (5-15 nm) using a flame spray method, the surface area of which was $\sim 25 \mathrm{~m}^{2} / \mathrm{g}, \sim 6$ times higher than that prepared using a conventional sol-gel method. On these nanoparticles, although the potential dependence of the XANES and EXAFS was subtle in a potential window of 1.20-1.55 V, an increase of Co average oxidation state and an increase of $\mathrm{CoO}(\mathrm{OH})$ contribution were clearly shown at OER potentials (1.55 V, Figure 3D-E). More obvious potential dependent changes were also observed on Co-Mn oxide[38] and Mn-doped cobalt ferrite ( 15 nm) [39], but over a much wider potential window (0.2-1.2 V). In addition, Fabbri et al.[37] also found that the oxidized Co atoms could not be reduced when the potential was stepped back to a low potential (1.2 V, Figure $3 F$ ), and they proposed that an oxy(hydroxide) surface layer was formed by irreversible surface reconstruction under OER condition. Such an activation process was supported by studies of CoFe spinels[40] and CoFe alloy nanoparticles[41], in which an amorphous layer was directly observed on the surface of the particles by electron microscopy after the catalysts were aged (the inset of Figure 3F). 

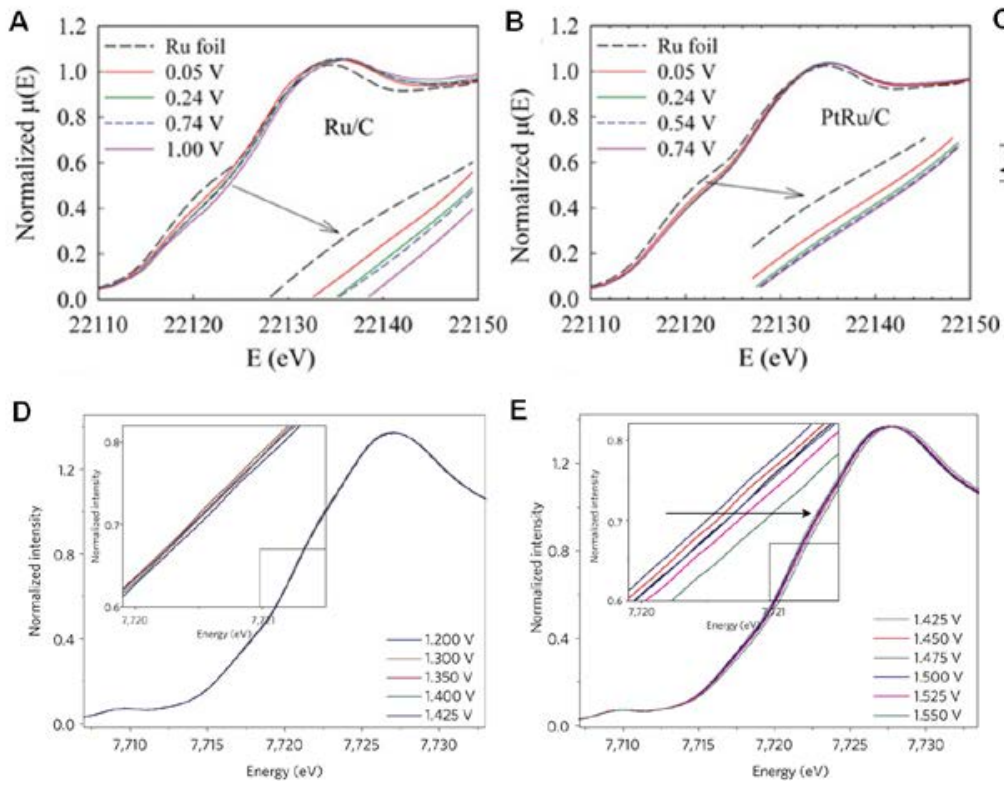

$E$

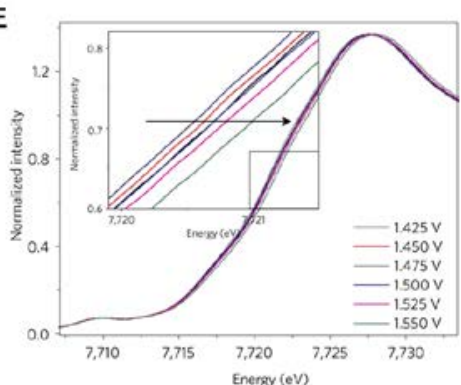

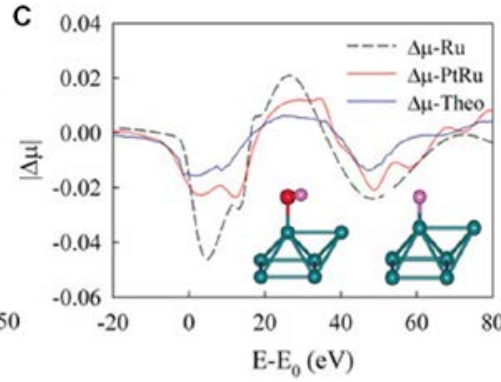

$\mathbf{F}$

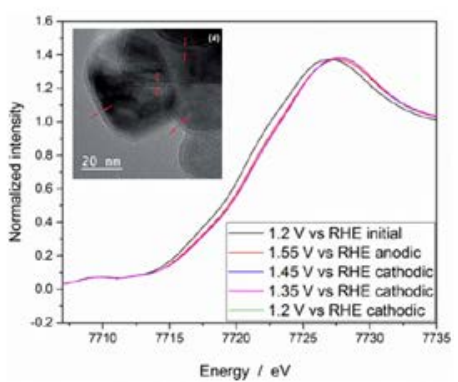

Figure $3(\mathrm{~A}-\mathrm{C})$ A $\Delta \mu$ method used to emphasise electrochemistry-induced changes on nanoparticles. Ru K edge XANES spectra of (A) Ru/C ( 8 nm) and (B) PtRu/C collected in $\mathrm{H}_{2}$-saturated 0.1 M KOH solution under potential control. (C) Comparison of experimental $\Delta \mu$ signals, derived from the XANES spectra in A and B $(\Delta \mu=\mu(0.24 \mathrm{~V})-\mu(0.05 \mathrm{~V}))$, with a theoretical $\Delta \mu$, modelled from clusters shown in the inset $\left(\Delta \mu\right.$-Theo $\left.=\mu\left(\mathrm{Ru}_{6}-\mathrm{OH}\right)-\mu\left(\mathrm{Ru}_{6}-\mathrm{H}\right)\right)$. Reproduced with permission from [31]. Copyright (2017) Wiley-VCH Verlag GmbH \& Co. KGaA, Weinheim. (D-F) Co K edge XANES spectra of a BSCF electrode in 0.1 M KOH during anodic potential holding (D) from 1.2 V to 1.425 V and (E) from 1.425 V to $1.55 \mathrm{~V}$. (F) Comparison of the Co K edge XANES spectra during cathodic potential holding from $1.55 \mathrm{~V}$ to $1.2 \mathrm{~V}$. Reprinted by permission from [37]. Copyright (2017) Springer Nature. (the inset of F) a post-mortem TEM image of an aged $\mathrm{FeCo}_{2} \mathrm{O}_{4}$ OER electrocatalyst, highlighting amorphous layers on the surface of particles. Reproduced with permission from [40], Copyright (2018) Royal Society of Chemistry.

\section{Core-shell nanoparticles and thin films - isolating the contribution of surface atoms}

Another strategy for in situ XAS studies of nanoparticles is to isolate the contribution of surface atoms from the core. For metallic nanoparticles, this may be accomplished by designing core-shell bimetallic particles or by depositing surface ad-atoms, wherein the element of interest is exclusively put on the surface of a second component, which may serve as an inert host or participate in structural or electronic modification of the surface atoms. Using this approach, the direct measurement of Pt surface oxidation using XAS can be achieved regardless of the particle size[42]. Sasaki et al.[25] prepared a Pt monolayer (ML) on Pd particles (4.2 nm, 35\% surface atoms) using the galvanic displacement of an underpotential deposited Cu ML. By linear combination fitting, they compared the oxide percentage of this $\mathrm{Pt}_{\mathrm{ML}} / \mathrm{Pd} / \mathrm{C}$ with that $\mathrm{Pt} / \mathrm{C}(2.6 \mathrm{~nm})$ per surface atoms 
(obtained using the fraction of surface atoms) as a function of applied potentials, and they found that the Pd core underneath significantly retarded the Pt oxidation.

The second component of Pt-based bimetallic electrocatalysts can also participate in electrochemical process. A good example is $\mathrm{PtRu} / \mathrm{C}$ catalysts for anodic reactions of fuel cells, the $\mathrm{Ru}$ site of which can be an adsorption site of $\mathrm{CO}$ intermediate and an active site for $\mathrm{OH}_{\text {ads }}$ formation at low potentials. Pelliccione et al.[43] compared the potential dependence of Ru K-edge spectra on a Ru@Pt catalyst (0.3 ML Ru on Pt nanoparticles, 10 nm) with or without the presence of methanol. In $0.1 \mathrm{M} \mathrm{H}_{2} \mathrm{SO}_{4}$, the $\mathrm{Ru}$ atoms were gradually oxidized with potential and started being transformed into $\mathrm{RuO}_{2}$-like species at $0.575 \mathrm{~V}$ (vs. Ag/AgCl). In the presence of methanol, however, the oxidation state and coordination environment of the $\mathrm{Ru}$ atoms were more stable without the formation of the $\mathrm{RuO}_{2}$-like species at high potentials but with additional Ru-CO species present at all the studied potentials. The authors attributed the different response to electrolyte to the dual role of $\mathrm{Ru}$ sites during methanol oxidation, providing $\mathrm{OH}_{\mathrm{ads}}$ species and available sites for $\mathrm{CO}_{\mathrm{ads}}$.

Similar to the core-shell approach, thin films of the active electrocatalyst can be deposited on to a substrate to minimise the contribution of the bulk. This method is particularly useful for the study of metal oxide electrocatalysts, prepared by electrodeposition or vapour deposition. In addition to the need to ensure electrochemical accessibility and activity of the full thickness of the film to enable accurate interpretation of the in situ XAS data, which is difficult for such metal-oxide films as conductivity is often an issue, attention must also be paid the presence of inactive or interfering phases (e.g. metal) from the deposition, which may contribute to the XAS signal and complicate the data interpretation.

Fe-containing $\mathrm{NiO}_{\mathrm{x}}$ and $\mathrm{CoO}_{\mathrm{x}}$ are regarded as highly active and nonprecious electrocatalysts for OER, even with a trace amount of Fe[44-46]. The promotional role of Fe and the incorporation between Fe and Ni were studied using in situ XAS measurements on electrodeposited Ni-Fe oxide thin films at both the $\mathrm{Ni} \mathrm{K}$ and the Fe K edge[47-49]. On all these thin films, The oxidation of $\mathrm{Ni}^{2+}$ to $\mathrm{Ni}^{3+}$ and the corresponding transformation of a $\alpha-\mathrm{Ni}(\mathrm{OH})_{2}$ phase to a $\gamma-\mathrm{NiOOH}$ phase were unambiguously detected by comparing spectra at the non-catalytic state $(1.23 \mathrm{~V})$ with that at catalytic state (1.55 V, Figure 4A and Figure 4C)[47], as evidenced by the positive shift of edge position and the shortened Ni-O in the first coordination shell and $\mathrm{Ni}-\mathrm{Ni} / \mathrm{Fe}$ bonds in the second coordination shell, the latter of which is characteristic of $\gamma$-NiOOH. The increase in the oxidation state of $\mathrm{Ni}$ (from 1.9 to 2.6) was less than that expected for the $\gamma$-NiOOH phase (nonstoichiometric, a mixture of $\mathrm{Ni}^{3+}$ and $\mathrm{Ni}^{4+}$ sites), which was due to the minor contribution of metallic $\mathrm{Ni}$ and the presence of a possible unreactive part of the thin film. For the Fe, a similar structural transition was 
also found by EXAFS (Figure 4D), with shorter Fe-O and Fe-M scattering paths, but the XANES spectra did not support the oxidation of $\mathrm{Fe}^{3+}$ (Figure 4B) [47]. The origin of the Fe local coordination changes was studied using time-resolved XAS fluorescence signals at fixed X-ray energies whilst conducting cyclic voltammetry or current transient measurements [47]. The fluorescence signal tracking the Ni oxidation state (chosen as the middle of the absorption edge, $8345 \mathrm{eV}$ ) and its derivative with time were found to match precisely with the CV current corresponding to the redox of the whole catalyst material (Figure $4 \mathrm{E}$ and $4 \mathrm{H}$ ), whilst such a potential dependence was not found at the Fe edge position (7126 eV) (Figure 4F). Alternatively, tracking the X-ray signal at $7131 \mathrm{eV}$ (the whiteline region of the Fe K-edge) during $\mathrm{CV}$ and potential step experiments revealed that the Fe coordination environment changed concurrently with the $\mathrm{Ni}^{2+} / \mathrm{Ni}^{3+}$ transition at the same redox potentials and with the same kinetics (Figure 4G and $4 \mathrm{H})$. The authors concluded that the redox current was associated exclusively with the $\mathrm{Ni}^{2+} / \mathrm{Ni}^{3+/ 4+}$ couple, which then induced the changes of the Fe coordination environment.

Drevon et al.[50] tracked the changes in the spectra of the electrodeposited Ni-Fe oxide thin film at the $\mathrm{O}$ K, Ni L and Fe L edges using the fixed energy cyclic voltammetry approach described above. In addition to similar potential dependences of the $\mathrm{Fe}$ and the $\mathrm{Ni}$ to that observed by González-Flores et al.[47], a new pre-peak at $529 \mathrm{eV}$ in the $\mathrm{O} \mathrm{K}$ edge spectra, assigned to the hybridization between $\mathrm{O}(2 \mathrm{p})$ and $\mathrm{Ni}(3 \mathrm{~d}) \mathrm{t}_{2 \mathrm{~g}}$ orbitals, was found. During a $\mathrm{CV}$ the $\mathrm{X}$-ray signal at this $\mathrm{O} \mathrm{K}$ pre-peak showed a nearly identical potential dependence with that at $\mathrm{Ni} \mathrm{L}_{3}$ shoulder reflecting the $\mathrm{Ni}$ redox transition (Figure $4 \mathrm{I}$ ), suggesting the appearance of the $\mathrm{O} \mathrm{K}$ pre-edge features was closely related to the oxidation of the $\mathrm{Ni}^{2+}$. By comparison with the literature and summarizing the metal oxidation observed in situ XAS, the authors proposed an OER mechanism for $\mathrm{Ni}-\mathrm{Fe}-\mathrm{O}$ catalysts and argued that the $\mathrm{O}$ species corresponding to the $\mathrm{O} \mathrm{K}$ pre-edge feature may be an electron deficient reactive $\mathrm{O}$ site, as an intermediate prior to O-O bond formation(Figure 4J). 

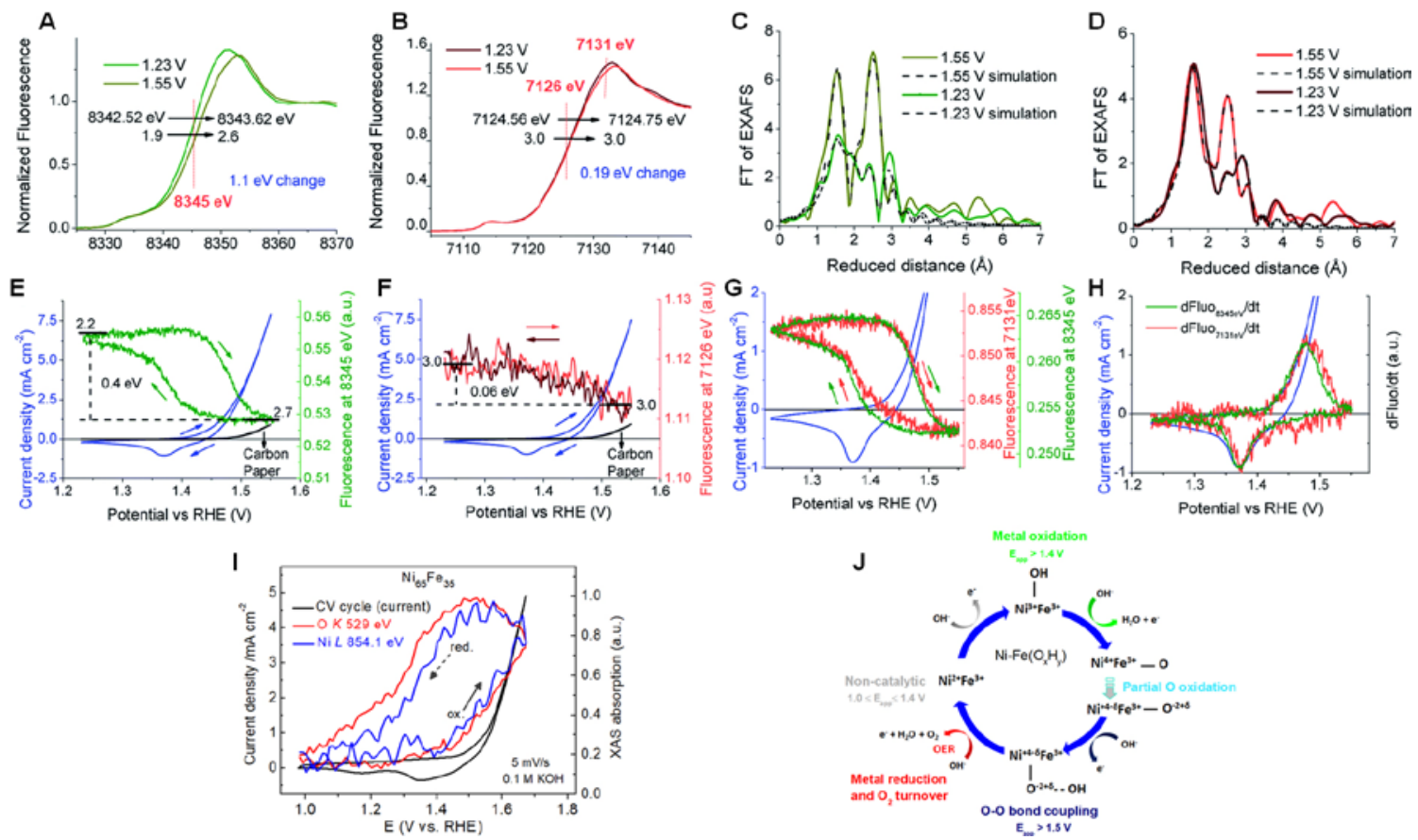

Figure 4 Time-resolved XAS signals of an electrodeposited Ni-Fe oxide thin film at fixed X-ray energies whilst conducting cyclic voltammetry in $1 \mathrm{M} \mathrm{KOH}$. (A and B) XANES spectra and (C and D) EXAFS spectra at $1.23 \mathrm{~V}$ and $1.55 \mathrm{~V}$ at (A and C) the Ni K edge and (B and D) the Fe K edge, showing the shift of edge positions (without correction of metallic Ni contribution using linear combination of reference compounds) and the energy at which the X-ray beam was set for the following time-resolved measurements (the red dash lines). Time-resolved detection of (E) Ni K-edge and (F) Fe K-edge XAS fluorescence signal at the respective edge positions during CV, showing the current density (blue), $\mathrm{X}$-ray fluorescence signal at $8345 \mathrm{eV}$ (green, for the $\mathrm{Ni} \mathrm{K}$ edge) and X-ray fluorescence signal at $7126 \mathrm{eV}$ (red, for the Fe K-edge). (G) X-ray fluorescence signals and (H) their derivative with time at $7131 \mathrm{eV}$ (orange, near the maximum of the Fe K white line) and at $8345 \mathrm{eV}$ (green, the Ni K edge) while recording a CV (blue). The CV measurements were carried out at a scan rate of $5 \mathrm{mV} \mathrm{s}^{-1}$, with $3 \Omega$ ohmic resistance from the electrolyte and electrodes. Adapted with permission from [47], Copyright (2018) Royal Society of Chemistry. (I) Time-resolved detection of O K-edge and Ni Ledge XAS transmission signal at fixed energy (854.1 eV for the Ni L-edge and $529 \mathrm{eV}$ for the O K-edge) during CV. The $\mathrm{CV}$ measurements were carried out in $0.1 \mathrm{M} \mathrm{KOH}$ with a scan rate of $5 \mathrm{mV} \mathrm{s}^{-1}$. (J) A proposed OER mechanism on NiFe-O electrocatalyst, summarizing the metal oxidation finding using in situ XAS measurements. Reproduced from the work of Drevon et al.[50], which is licensed under Creative Commons Licence 4.0 CC BY.

\section{Conclusions}

XAS conducted in situ or operando is a valuable tool to probe the dynamic electronic and coordination structure of electrocatalysts under working conditions, but care must be taken when designing the experiments. In this review, we presented recent contributions in which in situ XAS measurements were designed so as to match the information obtained from electrochemical measurement by tailoring the sample to ensure that the XAS data reflected the electrochemically 
active features of the sample, be that the metal atoms of single-metal atom electrocatalysts, the surface of metal nanoparticle electrocatalysts or the surface and near surface region of metal-oxide electrocatalysts. Such surface and near-surface sensitivity is achieved by decreasing the particle size, employing $\Delta \mu$ analysis, designing core-shell configurations, and carefully ensuring that the full thickness of the electrocatalyst sample is electrochemically accessible and active. Even when such careful experimental design is employed the inability to identify coordination to atoms with similar masses, especially the electrocatalytically important $\mathrm{C}, \mathrm{N}$, and $\mathrm{O}$ atoms, remains a problem when employing XAS methods to characterise electrocatalysts and electrocatalytic reactions. Further developments employing soft (lower energy) XAS at the $\mathrm{C}, \mathrm{N}$ or $\mathrm{O}$ edge or the use of new techniques such as resonant inelastic X-ray scattering (RIXS, or called X-ray Raman), which can provide information regarding the chemical bonding between the metal adsorber and the adsorbate, represent the next stages in the use of in situ or operando XAS to study electrocatalysts and electrocatalysis, although each of these will be accompanied by new challenges that must be overcome.

\section{Corresponding author}

*Andrea E. Russell.: e-mail: a.e.russell@soton.ac.uk.

\section{Author contribution}

The manuscript was written through contributions of all authors. All authors have given approval to the final version of the manuscript.

\section{Conflict of interest}

The authors declare no competing financial interests

\section{Acknowledgements}

HH acknowledges Fellowship support from the China Scholarship Council (201608440295). 


\section{References}

Papers of particular interest, published within the period of review, have been highlighted as:

* of special interest

** of outstanding interest

[1] J. Li, J. Gong, Operando characterization techniques for electrocatalysis, Energy Environ. Sci., 13 (2020) 3748-3779.

[2] M. Wang, L. Árnadóttir, Z.J. Xu, Z. Feng, In situ X-ray absorption spectroscopy studies of nanoscale electrocatalysts, Nano-Micro Lett., 11 (2019) 47.

[3] J.J. Velasco-Velez, R.V. Mom, L.E. Sandoval-Diaz, L.J. Falling, C.H. Chuang, D. Gao, T.E. Jones, Q. Zhu, R. Arrigo, B. Roldan Cuenya, A. Knop-Gericke, T. Lunkenbein, R. Schlogl, Revealing the Active Phase of Copper during the Electroreduction of $\mathrm{CO}_{2}$ in Aqueous Electrolyte by Correlating In Situ X-ray Spectroscopy and In Situ Electron Microscopy, ACS Energy Lett., 5 (2020) 2106-2111.

[4] E.A. Carbonio, J.-J. Velasco-Velez, R. Schlögl, A. Knop-Gericke, Perspective-Outlook on Operando Photoelectron and Absorption Spectroscopy to Probe Catalysts at the Solid-Liquid Electrochemical Interface, J. Electrochem. Soc., 167 (2020) 054509.

[5] M. Farmand, A.T. Landers, J.C. Lin, J.T. Feaster, J.W. Beeman, Y. Ye, E.L. Clark, D. Higgins, J. Yano, R.C. Davis, A. Mehta, T.F. Jaramillo, C. Hahn, W.S. Drisdell, Electrochemical flow cell enabling operando probing of electrocatalyst surfaces by X-ray spectroscopy and diffraction, Phys. Chem. Chem. Phys., 21 (2019) 5402-5408.

[6] N.J. Firet, M.A. Blommaert, T. Burdyny, A. Venugopal, D. Bohra, A. Longo, W.A. Smith, Operando EXAFS study reveals presence of oxygen in oxide-derived silver catalysts for electrochemical $\mathrm{CO}_{2}$ reduction, J. Mater. Chem. A, 7 (2019) 2597-2607.

[7] J. Han, J. Bian, C. Sun, Recent advances in single-atom electrocatalysts for oxygen reduction reaction, Research, 2020 (2020) 9512763.

[8] B. Lu, Q. Liu, S. Chen, Electrocatalysis of single-atom sites: impacts of atomic coordination, ACS Catal., 10 (2020) 7584-7618.

[9] R. Qin, K. Liu, Q. Wu, N. Zheng, Surface coordination chemistry of atomically dispersed metal catalysts, Chem. Rev., 120 (2020) 11810-11899.

[10] Q. Jia, N. Ramaswamy, U. Tylus, K. Strickland, J. Li, A. Serov, K. Artyushkova, P. Atanassov, J. Anibal, C. Gumeci, S.C. Barton, M.-T. Sougrati, F. Jaouen, B. Halevi, S. Mukerjee, Spectroscopic insights into the nature of active sites in iron-nitrogen-carbon electrocatalysts for oxygen reduction in acid, Nano Energy, 29 (2016) 65-82.

[11] Q. Jia, E. Liu, L. Jiao, S. Pann, S. Mukerjee, X-Ray absorption spectroscopy characterizations on PGM-free electrocatalysts: justification, advantages, and limitations, Adv. Mater., 31 (2019) e1805157.

[12] Q. Jia, N. Ramaswamy, H. Hafiz, U. Tylus, K. Strickland, G. Wu, B. Barbiellini, A. Bansil, E.F. Holby, P. Zelenay, S. Mukerjee, Experimental observation of redox-induced Fe-N switching behavior as a determinant role for oxygen reduction activity, ACS Nano, 9 (2015) 12496-12505. 
[13] J. Li, S. Ghoshal, W. Liang, M.-T. Sougrati, F. Jaouen, B. Halevi, S. McKinney, G. McCool, C. Ma, X. Yuan, Z.-F. Ma, S. Mukerjee, Q. Jia, Structural and mechanistic basis for the high activity of Fe-N-C catalysts toward oxygen reduction, Energy Environ. Sci., 9 (2016) 2418-2432.

[14] M. Xiao, J. Zhu, L. Ma, Z. Jin, J. Ge, X. Deng, Y. Hou, Q. He, J. Li, Q. Jia, S. Mukerjee, R. Yang, Z. Jiang, D. Su, C. Liu, W. Xing, Microporous framework induced synthesis of singleatom dispersed $\mathrm{Fe}-\mathrm{N}-\mathrm{C}$ acidic ORR catalyst and its in situ reduced $\mathrm{Fe}-\mathrm{N}_{4}$ active site identification revealed by X-ray absorption spectroscopy, ACS Catal., 8 (2018) 2824-2832.

[15] J. Li, A. Alsudairi, Z.F. Ma, S. Mukerjee, Q. Jia, Asymmetric volcano trend in oxygen reduction activity of Pt and non-Pt catalysts: in situ identification of the site-blocking effect, J. Am. Chem. Soc., 139 (2017) 1384-1387.

*[16] L. Osmieri, R.K. Ahluwalia, X. Wang, H.T. Chung, X. Yin, A.J. Kropf, J. Park, D.A. Cullen, K.L. More, P. Zelenay, D.J. Myers, K.C. Neyerlin, Elucidation of Fe-N-C electrocatalyst active site functionality via in-situ X-ray absorption and operando determination of oxygen reduction reaction kinetics in a PEFC, Appl. Catal., B, 257 (2019) 117929.

A quasi-operando XAS study of Fe-N-C at the membrane electrode assembly level supported the site-blocking mechanism of Fe- $\mathrm{N}_{4}$ for ORR proposed by Mukerjee’s group.

**[17] N. Zhang, T. Zhou, J. Ge, Y. Lin, Z. Du, C.a. Zhong, W. Wang, Q. Jiao, R. Yuan, Y. Tian, W. Chu, C. Wu, Y. Xie, High-density planar-like $\mathrm{Fe}_{2} \mathrm{~N}_{6}$ structure catalyzes efficient oxygen reduction, Matter, 3 (2020) 509-521.

The authors reported a new and efficient $\mathrm{Fe}_{2} \mathrm{~N}_{6}$ active site of $\mathrm{Fe}-\mathrm{N}-\mathrm{C}$ catalysts for ORR, facilitating the dual adsorption of $\mathrm{O}_{2}$ and its intermediates compared to the isolated $\mathrm{Fe}-\mathrm{N}_{4}$ active site. In situ XAS was used to study the $\mathrm{Fe}^{2+} / \mathrm{Fe}^{3+}$ redox and the contraction of the first-shell FeFe scattering path under electrochemical environment.

**[18] J. Gu, C.S. Hsu, L. Bai, H.M. Chen, X. Hu, Atomically dispersed Fe ${ }^{3+}$ sites catalyze efficient $\mathrm{CO}_{2}$ electroreduction to CO, Science, 364 (2019) 1091-1094.

In situ XAS was used to monitor the redox transition and the corresponding geometry behaviour of Fe-N-C catalysts as a function of applied potentials, which was found to vary significantly by changing the type of the $\mathrm{N}$ ligands from pyridinic $\mathrm{N}$ to pyrrolic $\mathrm{N}$.

**[19] H. Shang, W. Sun, R. Sui, J. Pei, L. Zheng, J. Dong, Z. Jiang, D. Zhou, Z. Zhuang, W. Chen, J. Zhang, D. Wang, Y. Li, Engineering isolated $\mathrm{Mn}-\mathrm{N}_{2} \mathrm{C}_{2}$ atomic interface sites for efficient bifunctional oxygen reduction and evolution reaction, Nano Lett., 20 (2020) 5443-5450.

The authors reported a bifunctional Mn- $\mathrm{N}_{2} \mathrm{C}_{2}$ electrocatalyst for ORR and OER, and in both potential ranges the in situ XAS showed potential dependence, with bond-length-elongated $\mathrm{Mn}^{2+}-\mathrm{N}_{2} \mathrm{C}_{2}$ during ORR and bond-length-contracted $\mathrm{Mn}^{4+}-\mathrm{N}_{2} \mathrm{C}_{2}$ during OER.

**[20] Z. Weng, Y. Wu, M. Wang, J. Jiang, K. Yang, S. Huo, X.F. Wang, Q. Ma, G.W. Brudvig, V.S. Batista, Y. Liang, Z. Feng, H. Wang, Active sites of copper-complex catalytic materials for electrochemical carbon dioxide reduction, Nat. Commun., 9 (2018) 415. 
In situ XAS was used to probe the interconvertible structural reconstruction of $\mathrm{CuPc}$ to $\mathrm{Cu}$ clusters during $\mathrm{CO}_{2} \mathrm{RR}$, and the formation of $\mathrm{Cu}$ clusters is correlated with the improved $\mathrm{CO}_{2^{-}}$ to- $\mathrm{CH}_{4}$ conversion.

[21] L.S. Ma, W.B. Hu, B.B. Mei, H. Liu, B. Yuan, J. Zang, T. Chen, L.L. Zou, Z.Q. Zou, B. Yang, Y. Yu, J.Y. Ma, Z. Jiang, K. Wen, H. Yang, Covalent triazine framework confined copper catalysts for selective electrochemical $\mathrm{CO}_{2}$ reduction: operando diagnosis of active sites, ACS Catal., 10 (2020) 4534-4542.

[22] D. Karapinar, N.T. Huan, N. Ranjbar Sahraie, J. Li, D. Wakerley, N. Touati, S. Zanna, D. Taverna, L.H. Galvao Tizei, A. Zitolo, F. Jaouen, V. Mougel, M. Fontecave, Electroreduction of $\mathrm{CO}_{2}$ on single-site copper-nitrogen-doped carbon material: selective formation of ethanol and reversible restructuration of the metal sites, Angew. Chem., Int. Ed., 58 (2019) 15098-15103.

*[23] H. Xu, D. Rebollar, H. He, L. Chong, Y. Liu, C. Liu, C.-J. Sun, T. Li, J.V. Muntean, R.E. Winans, D.-J. Liu, T. Xu, Highly selective electrocatalytic $\mathrm{CO}_{2}$ reduction to ethanol by metallic clusters dynamically formed from atomically dispersed copper, Nat. Energy, 5 (2020) 623-632.

In situ XAS was used to detect a reversible transformation of isolated $\mathrm{Cu}$ atoms to $\mathrm{Cu}_{3 \sim 4}$ clusters, and the authors correlated the formation of the $\mathrm{Cu}$ clusters to the high selectivity of $\mathrm{CO}_{2}$-toethanol conversion.

[24] A.E. Russell, A. Rose, X-ray absorption spectroscopy of low temperature fuel cell catalysts, Chem. Rev., 104 (2004) 4613-4636.

[25] K. Sasaki, N. Marinkovic, H.S. Isaacs, R.R. Adzic, Synchrotron-based in situ characterization of carbon-supported platinum and platinum monolayer electrocatalysts, ACS Catal., 6 (2015) 69-76.

[26] H. Imai, K. Izumi, M. Matsumoto, Y. Kubo, K. Kato, Y. Imai, In situ and real-time monitoring of oxide growth in a few monolayers at surfaces of platinum nanoparticles in aqueous media, J. Am. Chem. Soc., 131 (2009) 6293-6300.

[27] L.R. Merte, F. Behafarid, D.J. Miller, D. Friebel, S. Cho, F. Mbuga, D. Sokaras, R. AlonsoMori, T.-C. Weng, D. Nordlund, A. Nilsson, B. Roldan Cuenya, Electrochemical oxidation of size-selected Pt nanoparticles studied using in situ high-energy-resolution X-ray absorption spectroscopy, ACS Catal., 2 (2012) 2371-2376.

[28] D.E. Ramaker, D.C. Koningsberger, The atomic AXAFS and $\Delta \mu$ XANES techniques as applied to heterogeneous catalysis and electrocatalysis, Phys. Chem. Chem. Phys., 12 (2010) 5514-5534.

[29] M. Teliska, W.E. O'Grady, D.E. Ramaker, Determination of O and OH adsorption sites and coverage in situ on Pt electrodes from $\mathrm{Pt}_{2,3}$ X-ray absorption spectroscopy, J. Phys. Chem. B, 109 (2005) 8076-8084.

*[30] Q. Jia, Z. Zhao, L. Cao, J. Li, S. Ghoshal, V. Davies, E. Stavitski, K. Attenkofer, Z. Liu, M. Li, X. Duan, S. Mukerjee, T. Mueller, Y. Huang, Roles of Mo surface dopants in enhancing the ORR performance of octahedral PtNi nanoparticles, Nano Lett., 18 (2018) 798-804.

An in situ XAS study of a Mo-doped PtNi/C octahedral catalyst for ORR. The Mo ad-atoms were found to be electrochemical active, with the $\mathrm{Mo}^{4+} / \mathrm{Mo}^{6+}$ redox transition at $0.45 \mathrm{~V}$, and 
the $\triangle \mathrm{XANES}$ at the $\mathrm{Pt} \mathrm{L}_{3}$ edge suggested that the oxygen coverage on Pt surface decreased when the Mo ad-atoms were present.

[31] J. Li, S. Ghoshal, M.K. Bates, T.E. Miller, V. Davies, E. Stavitski, K. Attenkofer, S. Mukerjee, Z.F. Ma, Q. Jia, Experimental proof of the bifunctional mechanism for the hydrogen oxidation in alkaline media, Angew. Chem., Int. Ed., 56 (2017) 15594-15598.

[32] C.S. Spanjers, T.P. Senftle, A.C. van Duin, M.J. Janik, A.I. Frenkel, R.M. Rioux, Illuminating surface atoms in nanoclusters by differential X-ray absorption spectroscopy, Phys. Chem. Chem. Phys., 16 (2014) 26528-26538.

[33] S. Maniguet, R.J. Mathew, A.E. Russell, EXAFS of carbon monoxide oxidation on supported Pt fuel cell electrocatalysts, J. Phys. Chem. B, 104 (2000) 1998-2004.

[34] C.F.J. König, J.A. van Bokhoven, T.J. Schildhauer, M. Nachtegaal, Quantitative analysis of modulated excitation X-ray absorption spectra: enhanced precision of EXAFS fitting, J. Phys. Chem. C, 116 (2012) 19857-19866.

[35] G.L. Chiarello, D. Ferri, Modulated excitation extended X-ray absorption fine structure spectroscopy, Phys. Chem. Chem. Phys., 17 (2015) 10579-10591.

[36] P. Müller, I. Hermans, Applications of modulation excitation spectroscopy in heterogeneous catalysis, Ind. Eng. Chem. Res., 56 (2017) 1123-1136.

[37] E. Fabbri, M. Nachtegaal, T. Binninger, X. Cheng, B.J. Kim, J. Durst, F. Bozza, T. Graule, R. Schaublin, L. Wiles, M. Pertoso, N. Danilovic, K.E. Ayers, T.J. Schmidt, Dynamic surface self-reconstruction is the key of highly active perovskite nano-electrocatalysts for water splitting, Nat. Mater., 16 (2017) 925-931.

[38] Y. Yang, Y. Wang, Y. Xiong, X. Huang, L. Shen, R. Huang, H. Wang, J.P. Pastore, S.H. Yu, L. Xiao, J.D. Brock, L. Zhuang, H.D. Abruna, In situ X-ray absorption spectroscopy of a synergistic Co-Mn oxide catalyst for the oxygen reduction reaction, J. Am. Chem. Soc., 141 (2019) 1463-1466.

[39] Y. Xiong, Y. Yang, X. Feng, F.J. DiSalvo, H.D. Abruna, A strategy for increasing the efficiency of the oxygen reduction reaction in Mn-doped cobalt ferrites, J. Am. Chem. Soc., 141 (2019) 4412-4421.

[40] L. Calvillo, F. Carraro, O. Vozniuk, V. Celorrio, L. Nodari, A.E. Russell, D. Debellis, D. Fermin, F. Cavani, S. Agnoli, G. Granozzi, Insights into the durability of Co-Fe spinel oxygen evolution electrocatalysts via operando studies of the catalyst structure, J. Mater. Chem. A, 6 (2018) 7034-7041.

[41] S. Song, J. Zhou, X. Su, Y. Wang, J. Li, L. Zhang, G. Xiao, C. Guan, R. Liu, S. Chen, H.-J. Lin, S. Zhang, J.-Q. Wang, Operando X-ray spectroscopic tracking of self-reconstruction for anchored nanoparticles as high-performance electrocatalysts towards oxygen evolution, Energy Environ. Sci., 11 (2018) 2945-2953.

[42] D. Friebel, V. Viswanathan, D.J. Miller, T. Anniyev, H. Ogasawara, A.H. Larsen, C.P. O'Grady, J.K. Norskov, A. Nilsson, Balance of nanostructure and bimetallic interactions in Pt model fuel cell catalysts: in situ XAS and DFT study, J. Am. Chem. Soc., 134 (2012) 9664-9671.

[43] C.J. Pelliccione, E.V. Timofeeva, J.P. Katsoudas, C.U. Segre, In situ Ru K-edge X-ray absorption spectroscopy study of methanol oxidation mechanisms on model submonolayer ru on Pt nanoparticle electrocatalyst, J. Phys. Chem. C, 117 (2013) 18904-18912. 
[44] F. Song, L. Bai, A. Moysiadou, S. Lee, C. Hu, L. Liardet, X. Hu, Transition metal oxides as electrocatalysts for the oxygen evolution reaction in alkaline solutions: an application-inspired renaissance, J. Am. Chem. Soc., 140 (2018) 7748-7759.

[45] L.-A. Stern, X. Hu, Enhanced oxygen evolution activity by $\mathrm{NiO}_{x}$ and $\mathrm{Ni}(\mathrm{OH})_{2}$ nanoparticles, Faraday Discuss., 176 (2014) 363-379.

[46] L. Trotochaud, S.L. Young, J.K. Ranney, S.W. Boettcher, Nickel-iron oxyhydroxide oxygenevolution electrocatalysts: the role of intentional and incidental iron incorporation, J. Am. Chem. Soc., 136 (2014) 6744-6753.

**[47] D. González-Flores, K. Klingan, P. Chernev, S. Loos, M.R. Mohammadi, C. Pasquini, P. Kubella, I. Zaharieva, R.D.L. Smith, H. Dau, Nickel-iron catalysts for electrochemical water oxidation - redox synergism investigated by in situ X-ray spectroscopy with millisecond time resolution, Sustainable Energy Fuels, 2 (2018) 1986-1994.

A fixed energy XANES-CV study of a mixed Ni-Fe oxide thin film to track the potential dependence of $\mathrm{Ni}$ and $\mathrm{Fe}$ in terms of oxidation state and local coordination.

[48] D. Friebel, M.W. Louie, M. Bajdich, K.E. Sanwald, Y. Cai, A.M. Wise, M.J. Cheng, D. Sokaras, T.C. Weng, R. Alonso-Mori, R.C. Davis, J.R. Bargar, J.K. Norskov, A. Nilsson, A.T. Bell, Identification of highly active Fe sites in (Ni,Fe)OOH for electrocatalytic water splitting, J. Am. Chem. Soc., 137 (2015) 1305-1313.

[49] F. Song, M.M. Busch, B. Lassalle-Kaiser, C.S. Hsu, E. Petkucheva, M. Bensimon, H.M. Chen, C. Corminboeuf, X. Hu, An unconventional iron nickel catalyst for the oxygen evolution reaction, ACS Cent. Sci., 5 (2019) 558-568.

**[50] D. Drevon, M. Gorlin, P. Chernev, L. Xi, H. Dau, K.M. Lange, Uncovering the role of oxygen in $\mathrm{Ni}-\mathrm{Fe}\left(\mathrm{O}_{\mathrm{x}} \mathrm{H}_{\mathrm{y}}\right)$ electrocatalysts using in situ soft X-ray absorption spectroscopy during the oxygen evolution reaction, Sci. Rep., 9 (2019) 1532.

A fixed energy soft-XAS-CV study of a mixed Ni-Fe oxide thin film to track the structural activation process for OER from perspective of $\mathrm{Ni}, \mathrm{Fe}$ and $\mathrm{O}$ at the same time. 